\title{
Foreword Observing the mm Universe with the NIKA2 camera
}

The mm Universe conference has brought together the scientific community working with the NIKA2 camera recently installed at the 30-m telescope of IRAM (Institut de RadioAstronomie Millimétrique) located in Pico Veleta (Spain). The conference has covered theoretical, observational and instrumental topics related to the mm Universe, i.e. from stellar to cosmological scales. It has been organized in Grenoble by the LPSC (CNRS, Université Grenoble Alpes) in June 2019. It was opened to the whole scientific community, i.e. NIKA2 collaboration members, open-time users and researchers interested by high-resolution $\mathrm{mm}$ observations. The number of participants was 75 , from 8 countries. The conference lasted one week with 55 presentations in plenary session ${ }^{1}$. This conference was dedicated to the first results obtained with the NIKA2 camera. It was the first edition in a series of conferences that will accompany the scientific exploitation of NIKA2 in the next decade.

The observation of the sky at millimetre wavelengths in the past years contributed to tremendous improvements in our understanding of a great variety of scientific topics ranging from the planetary and star formation in the Milky Way to the measurement of cosmological parameters. The advent of the NIKA2 camera is a follow-up of the Planck and Herschel satellites that continues to reveal the details of the formation and evolution of structures throughout the Universe. NIKA2 is a millimetre camera, capable of surveying large areas of the sky at a high-angular resolution, with a high sensitivity and a large field of view. It has been designed and built by the NIKA2 collaboration and installed in October 2015 at the IRAM 30-m telescope. NIKA2 is open to the scientific community for observations for the next decade. NIKA2 will provide key observations from the galactic scale to the high redshift Universe. It will allow observers to address questions, such as the environment impact on dust properties, the star formation processes at low and high redshifts, the evolution of the large-scale structures and the use of galaxy clusters for precision cosmology.

The conference provided a stimulating forum for discussions on scientific topics related to NIKA2: instruments, data analysis, latest results and implications, upgrades and forthcoming projects. It was a great success thanks to the enthusiasm of the participants and the high quality of their presentations. The organization of the conference was made possible thanks to funding from the ANR (French National Research Agency) under the contrat ANR-15-CE31-0017 (NIKA2Sky). It was also partially funded by the LabEx FOCUS (ANR-11- LABX-0013) and the LPSC.

All proceedings articles in this volume have been peer-reviewed. All referees and authors should be thanked for their contribution to the editing process of the mm Universe conference proceedings.

F. Mayet (chair), A. Catalano, J. F. Macías-Pérez and L. Perotto

Scientific editors of the conference "mm Universe @ NIKA2"

\footnotetext{
${ }^{1}$ see https://lpsc-indico.in2p3.fr/Indico/event/1765
} 